\title{
Applicability of the P19CL6 cells as a model of cardiomyocytes - a transcriptome analysis
}

\author{
Iraj Khodadadi ${ }^{1,2}$, Nick J. Plant ${ }^{2}$, Vassilis Mersinias ${ }^{3}$, Alfred E. Thumser ${ }^{{ }^{\star}}$ \\ ${ }^{1}$ Department of Biochemistry and Nutrition, Hamedan University of Medical Sciences, Hamedan, Iran \\ ${ }^{2}$ Division of Biochemical Sciences, University of Surrey, Guildford, United Kingdom \\ ${ }^{3}$ B.S.R.C. "Alexander Fleming”, Varkiza, Greece; a.thumser@surrey.ac.uk
}

Received 15 November 2009; revised 4 December 2009; accepted 7 December 2009.

\begin{abstract}
The P19CL6 cell-line, a clone of the P19 mouse embryonal carcinoma cell-line, has been extensively used as a model for cardiomyocytes as these cells can be differentiated into a cardiomyocyte phenotype upon incubation with dimethyl sulfoxide. Uniquely, these cells can be observed to "beat" when monitored by microscopy. We started investigating the response of P19CL6 cells to fatty acids, but highly variable results lead us to investigate the phenotype of the P19CL6 cells in more depth. In this study we demonstrated that the P19CL6 cells are responsive to adrenaline, but loose the "beating" phenotype after 16 passages in culture. Analysis of specific mRNA transcripts indicated that the P19CL6 cells express both cardiac- and skeletal muscle-specific genes, while global analysis of microarray data showed clear differences between the P19CL6 cells and cardiac tissue of embryonic or adult origin. In conclusion, our observations suggest caution in the use of the P19CL6 cells as a model of cardiomyocytes unless rigorous validation for the intended analysis has been undertaken.
\end{abstract}

Keywords: Gene expression; Cardiomyocyte; P19CL6 Cell-line

\section{INTRODUCTION}

There is a requirement for the development of realistic cell culture models both for basic research and the development of novel therapeutic agents. However, for several tissues, including heart, no individual cell line has been successfully validated for these purposes; such a failure is usually the result of the loss of one or more specific phenotypic features associated with the target tissue in the cell line. One approach to mitigate these issues has been the utilisation of chemically-stimulated differentiation of stem cells, with the hope that these cell lines will have a more realistic phenotype than cell lines derived from fully differentiated tissue. Pluripotent embryonic carcinoma cells have been reported as successful in vitro models of cardiac differentiation; for example, the P19 mouse embryonal carcinoma cell-line has been reported to differentiate into an embryonic cardiac-muscle phenotype in vitro [1] upon the addition of dimethyl sulfoxide (DMSO) [2-5]. Differentiated P19 cells have been reported to retain the ability to spontaneously contract and shown to express transcripts in a temporal manner during culture, suggestive of a cardiac-muscle phenotype [5-7], and as such these cells have therefore been extensively used to study cardiac cell physiology $[1,2,5,6,8]$, although with the caveat that these cells are embryonic instead. However, in addition to these cardiacmuscle-specific properties, P19 cells also display pluripotent properties and can be differentiated into cells displaying either a skeletal muscle or neural phenotype [1, 3-5]. There has thus been some concern about the homogeneity of DMSO-differentiated P19 cultures, with a heterogeneous cell population following differentiation significantly reducing the utility of these cells as a cardiac-muscle-specific model: There has thus been much interest in identifying subclones of P19 cells that more robustly differentiate into cardiomyocytes. The P19CL6 cell-line, a sub-clone of P19 embryonal cells, has been reported to efficiently differentiate into beating cardiomyocytes upon exposure to DMSO under adherent culture conditions [9] and has been widely used as an in vitro model of cardiovascular cells $[1,2,10-16]$.

It is clear that the P19CL6 cell line has potential as a model system for the study of cardiomyocyte development and differentiation, and indeed they are currently used as such. However, full characterisation and validation is required before they can be used for this purpose with full confidence. A review of the literature, focusing on P19CL6 and P19 cell culture conditions, shows that two separate methods are commonly used, namely adherent and non-adherent culture conditions $[1,5,9,10,17]$. 
In addition, vitamins and hormones such as adrenaline have been shown to act as potent inducers of P19 cell differentiation into cardiomyocytes in addition to the aforementioned DMSO $[1,5,7,9]$. In this study we have characterized the P19CL6 cells in more detail under different culture conditions, focusing in particular on utilising microarray methodologies to compare the P19CL6 transcriptome against native cardiac-muscle and skeletal-muscle transcriptomes. These investigations represent the first robust examination of both P19CL6 transcriptome and cardiac phenotype, and demonstrate that the P19CL6 cell-line displays only a limited cardiomyocyte phenotype that is dependent on passage conditions. As such we would advise caution in the use of this cell line as a 'complete' in vitro model of cardiac-muscle cells.

\section{MATERIALS AND METHODS}

\subsection{Materials}

Cell culture media and reagents were obtained from Invitrogen Corporation (Paisley, U.K.) and Sigma-Aldrich Company Ltd. (Poole, U.K.). Materials and kits for RNA extraction, cDNA synthesis and RT-PCR were supplied by Promega Corporation (Southampton, U.K.), Amersham Biosciences (Chalfont St. Giles, U.K.) and Qiagen Ltd. (Crawley, U.K.). Corning Life Sciences (Schiphol-Rijk, Netherlands) supplied the ProntoPlus microarray kit. Ambion Ltd. (Huntingdon, U.K.) supplied mouse heart and embryonic total-RNA, whereas mouse skeletal muscle and embryonic heart total-RNA were purchased from Panomics Inc. (Redwood City, U.S.A.) and Zyagen (San Diego, U.S.A.), respectively.

\subsection{Cell culture}

P19CL6 cells were purchased at passage 9 from Riken Cell Bank (Ibaraki, Japan) in growing flask and cultured in medium containing $\alpha$-MEM (minimal essential media) supplemented with 10\% FBS (foetal bovine serum) and $1 \%$ penicillin-streptomycin $(10,000$ Units/ml and 10 $\mathrm{mg} / \mathrm{ml}$, respectively) [9]. To differentiate P19CL6 cells into cardiomyocytes, cells were plated in 6-well culture plates $\left(10 \mathrm{~cm}^{2}\right)$ at a density of $2 \times 10^{4}$ cells $/ \mathrm{cm}^{2}$ in standard medium containing 1\% DMSO [9]. Cells were cultured for 15 days at $37^{\circ} \mathrm{C}$ and $5 \% \mathrm{CO}_{2}$ with medium refreshed every second day. For culturing P19CL6 cells under non-adherent conditions, cells were stimulated to form aggregates by incubation in bacterial petri dishes $\left(1 \times 10^{6}\right.$ cells/dish; $78 \mathrm{~cm}^{2}$ ), containing a thin layer of $0.5 \%$ agar, for 4 days with standard media containing 1\% DMSO, before transfer to regular cell culture flasks for the remainder on the incubation period [5]. Cell aggregates were collected by centrifugation and replated into culture flasks for 15 days at $37^{\circ} \mathrm{C}$ and $5 \% \mathrm{CO}_{2}$ in the presence of $1 \%$ DMSO. The H9C2 (2-1) cell-line, a murine cell-line that expresses a skeletal muscle phenotype, was obtained from the European Collection of Cell Cultures (ECACC; Salisbury, U.K.) and cultured under the same adherent conditions as the P19CL6 cells.

\subsection{Determination of mRNA Levels by Reverse Transcriptase Polymerase Chain Reaction (RT-PCR) and cDNA Microarrays}

Total RNA was isolated from cells with Trizol reagent, as per manufacturer's instructions (Invitrogen Corporation, Paisley, U.K.). Whole heart and skeletal muscle tissues (upper leg muscle) were dissected from 10 week old male CD1 wild-type mice $(+/+)$, homogenised in Trizol reagent using an Ultra-Turrax T8 homogeniser, and total RNA isolated as per manufacturer's instructions. Gene-specific forward and reverse primers used in the one-step RT-PCR or nested PCR reactions are shown in Table 1. In samples that did not show detectable cDNA levels after an initial RT-PCR amplification the PCR products were reamplified by nested PCR [18]. PCR products were separated by electrophoresis on $2 \%$ agarose gels containing ethidium bromide, and the identity of PCR products verified by sequencing.

Microarray experiments were designed as dual-hybridisation optimal interwoven loops (http://exgen.ma.umist.ac.uk) [19-21]. cDNA was synthesised from purified total- RNA and labelled by a direct labelling method in the presence of Oligo dT, nucleotide mixture, Cy3-/Cy5- dCTP dyes, and SuperScript-II Reverse Transcriptase, based on Human Genome Mapping Project protocols (http://www.hgmp.mrc.ac.uk/). Purified Cy3- and Cy5- labelled cDNA samples (40 pmol each) were mixed in pairs (total volume $40 \mu \mathrm{l}$ ), according to the experimental plan, and hybridised to microarrays by incubation in hybridization chambers for 20 hours at $50^{\circ} \mathrm{C}$, before post-hybridization washing and drying. Microarray images were acquired using an Affymetrix 428 scanner (Affymetrix, Santa Clara, USA) and analysed with BlueFuse (BlueGnome Ltd., Cambridge, U.K.), including quantification of pixel intensities of the spots and excluding background intensity and artefact areas on the arrays. The data was filtered by eliminating low quality array spots, as determined by BlueFuse's spot uniformity and circularity measurements (spot uniformity and circularity $>0.5$ in at least $50 \%$ of the arrays), and normalised by intensity-dependent per spot and per chip (LOWESS) normalisation with GeneSpring-7 data analysis software (Agilent Technologies UK Ltd, Stockport, UK).

After Lowess normalisation and filtering of microarray data linear modelling (LLAMA; Live Linear Analysis of MicroArray) was performed (http://exgen.ma.umist.ac.uk) to convert data from the loop experiment into a linear model and generate differential gene expression estimates 


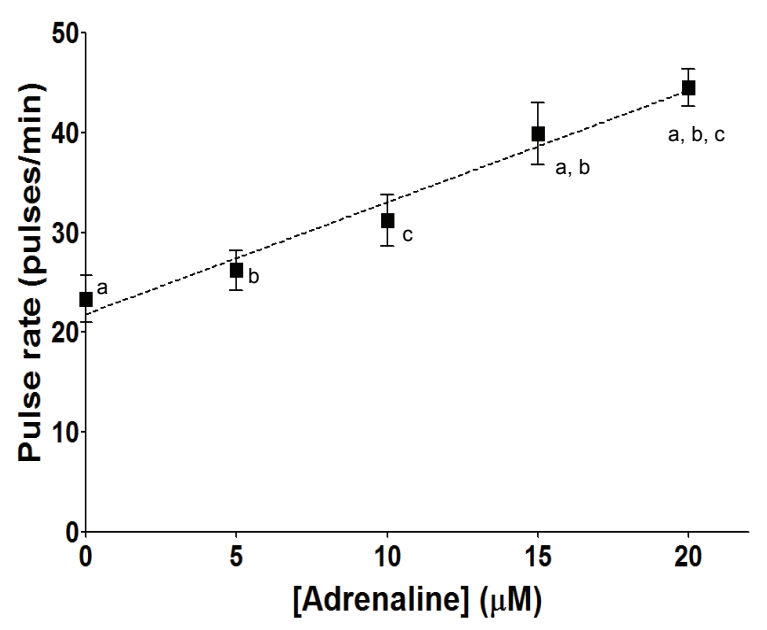

(a)

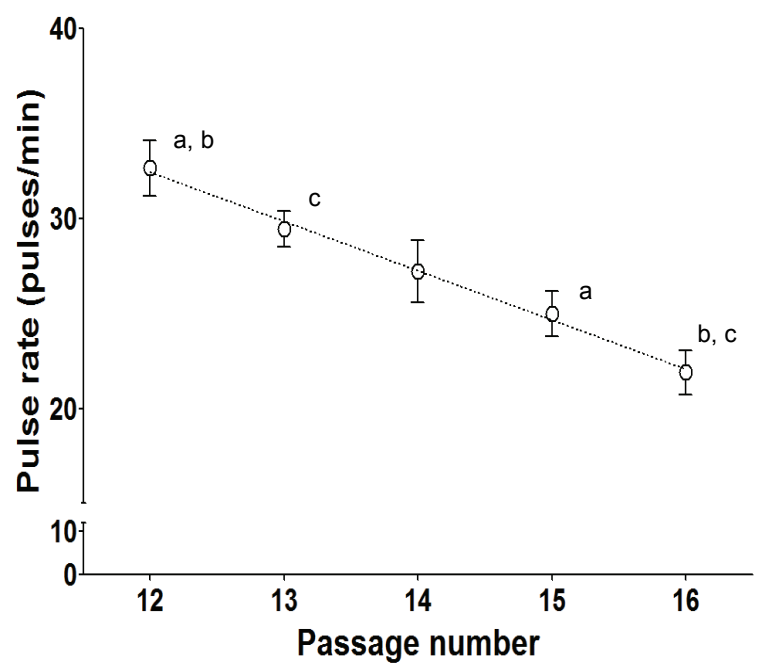

(b)

Figure 1. Determination of the effect of (a) passage number and (b) adrenaline on the beating characteristic in P19CL6 cells cultured under adherent conditions. (a) P19CL6 cells (passage 12) were cultured with $0-20 \mu \mathrm{M}$ adrenaline and pulse rate was recorded on day 15 of incubation. Linear regression analysis showed a correlation of pulse rate with adrenaline concentration $\left(\mathrm{y}=1.1 \mathrm{x}+21.8, \mathrm{R}^{2}=0.541, \mathrm{P}<\right.$ 0.001 ) and one-way ANOVA confirmed a significant effect of adrenaline on pulse rate in P19CL6 cells $(\mathrm{P}<0.001)$. Cells were cultured in three biological repeats and beating was recorded in at least six localized areas in the well. a, b, and c represent groups with a significant difference in average pulse rate. (b) P19CL6 cells from different passage numbers (12-16) were cultured in the presence of DMSO and pulse rate (beats/min) was recorded on day 15 of incubation. Linear regression analysis shows a significant effect of passage number on pulse rate $\left(\mathrm{y}=-2.58 \mathrm{x}+63.3, \mathrm{R}^{2}=0.486, \mathrm{P}<\right.$ 0.001 ) and two-way ANOVA confirmed significance difference between the different passage numbers of P19CL6 cells $(\mathrm{P}<0.01)$.

between contrast pairs, i.e. $\mathrm{Cy} 3 / \mathrm{Cy} 5$ ratios $[20,21,33]$. The
P19CL6 samples consisted of three biological repeats, with each sample analyzed on at least three microarray slides (technical repeats), and these were reduced to a single sample during the LLAMA analysis. The data reduction generated by the LLAMA analysis was further evaluated by PCA (principal component analysis) and PLS (partial least squares regression) (Simca-P+, Umetrics, New Jersey, U.S.A.). The PCA analysis generated correlations between the different samples. In order to simplify the interpretation of the PLS analysis, the P19CL6 sample was compared to skeletal muscle, adult and embryonic heart samples, with the H9C2 (2-1) sample removed. The PLS analysis was used to produce a variable importance (VIP) list and all genes with a VIP $>1$, i.e. having a significant impact on the difference between the P19CL6 and other samples, were further analysed with the DAVID software suite (http://david.abcc.ncifcrf.gov/home.jsp) for functional annotation clustering according to their biological functions [24].

\section{RESULTS}

In a series of separate cultures of P19CL6 cells it was noted that the cultures did not consistently display a "beating" phenotype, a characteristic that has previously been used as evidence of the cardiomyocyte properties of this P19 sub-clone [9].

\subsection{P19CL6 Cells Exhibit a Cardiac-Muscle-Like 'Beating' Phenotype under Specified Culture Conditions}

A characteristic of the P19CL6 cells is that they display beating in localised nodes following differentiation in the presence of DMSO [9]. The cardio-stimulatory chemical adrenaline elicited a dose-dependent linear increase in the observed pulse rate with statistically significant increases observed between 0-20 $\mu \mathrm{M}$ adrenaline (Figure 1a). However, observation of P19CL6 cells over time in culture (passages 12-22) demonstrated that beating was consistently only observed between passages $12-16$, and quantification of this beating showed a significant inverse correlation of the pulse-rate with passage number (Figure 1b). Microscopic analysis of DMSO-differentiated P19CL6 cells demonstrated mono-nuclear cells with no evidence of cell fusion; these characteristics were distinct from the morphological characteristics of skeletal muscle cells, as typified by the H9C2 (2-1) murine cell-line (Figure 2), and added further weight to the assertion that differentiated P19CL6 cells exhibit a cardiac-specific muscle phenotype $[5,10,34]$. The microscopic analysis and response to adrenaline provided an indication of a cardiac-type phenotype, although the loss of beating with passage number suggested that the phenotype may not be robust with regards to culture duration. 

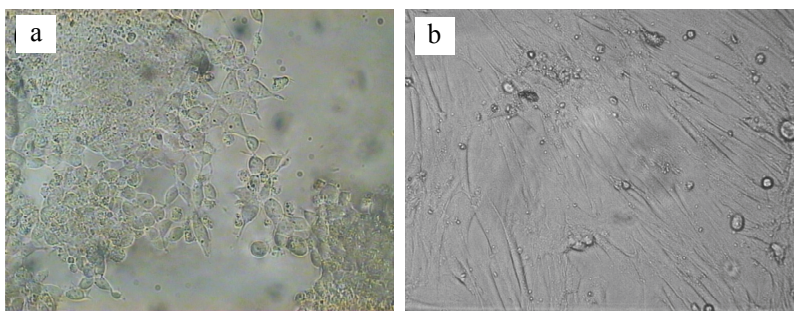

Figure 2. Microscopic examination of P19CL6 and H9C2(2-1) cells. Cells were cultured on coverslips under adherent conditions in the presence of DMSO and collected on day 15 of incubation. Cells were fixed using paraformaldehyde and observed by light microscopy under $40 \times$ magnification. (a) P19CL6 cells; (b) H9C2(2-1) cells.

\subsection{The P19CL6 Cell-Line Expresses both Cardiac- and Skeletal Muscle-Specific Transcripts}

To further examine the reported cardiac properties of the P19CL6 cell-line, mRNA transcript levels of $\alpha$-MHC, $\beta$ MHC, MyoD and myogenin were determined by RTPCR followed by nested PCR. The expression of these gene products are characteristic of cardiac- $(\alpha-\mathrm{MHC}$, $\beta$-MHC) and skeletal muscle (MyoD, myogenin) tissues [35-38]. Figure 3a shows that P19CL6 cells expressed significant levels of $\alpha-\mathrm{MHC}, \beta-\mathrm{MHC}, \mathrm{MyoD}$ and myogenin transcripts, inconsistent with a cardiac only phenotype: To examine if the unexpected expression of skeletal muscle markers in P19CL6 cells was caused by the culture conditions we also examined P19CL6 under non-adherent culturing conditions; under these conditions we once again observed both cardiac- and skeletal muscle-specific markers (Figure 3b), suggestive of a mixed cardiac/skeletal-muscle transcriptome in P19CL6 cells regardless of culture conditons. The identity of the $\alpha$ MHC, $\beta$-MHC, MyoD and myogenin transcripts was confirmed by sequencing of the nested PCR products (data not shown). The marker transcript specificity was confirmed using both the H9C2 (2-1) cell-line, which expresses a skeletal muscle phenotype, and only expressed MyoD and myogenin transcripts and mouse cardiac tissue, which only expressed the aforementioned transcript markers of cardiac phenotype, $\alpha-\mathrm{MHC}$ and $\beta$-MHC (Figure 3c). These data were thus consistent with P19CL6 cells exhibiting a mixed cardiac/skeletal muscle phenotype, and therefore a global transcriptome analysis was under-taken to examine this hypothesis, comparing P19CL6 cells with the H9C2 (2-1) cell-line and mouse cardiac and skeletal muscle tissue.

\subsection{Characterisation of the P19CL6 Cell-Line by Microarray Analysis}

Transcriptomes from three independent P19CL6 cultures were compared by microarray analysis to the transcript-

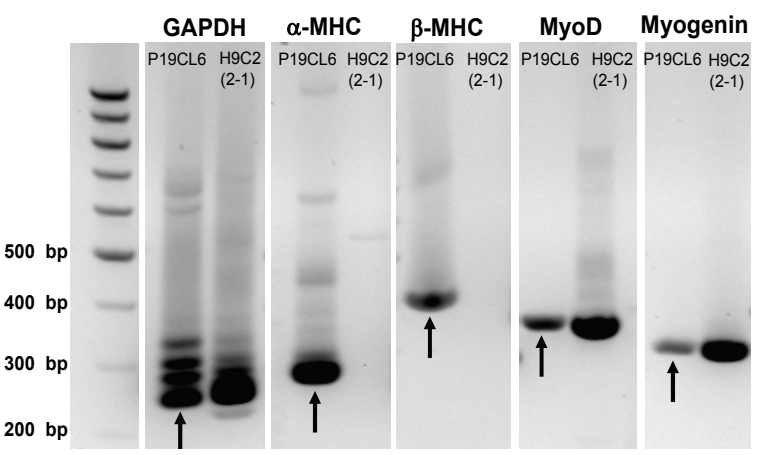

(a)

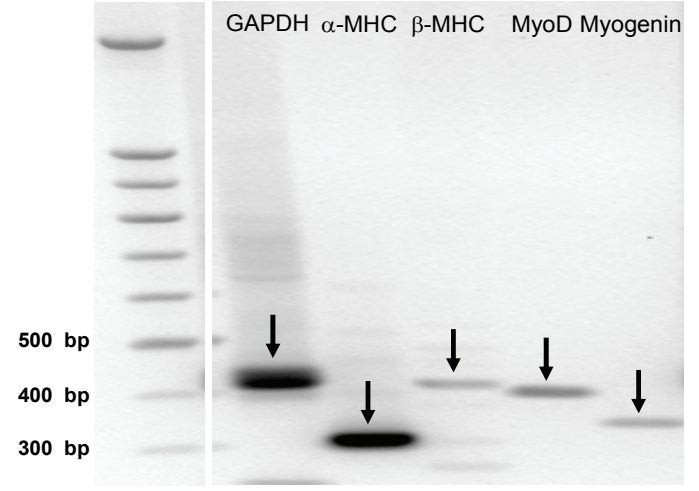

(b)

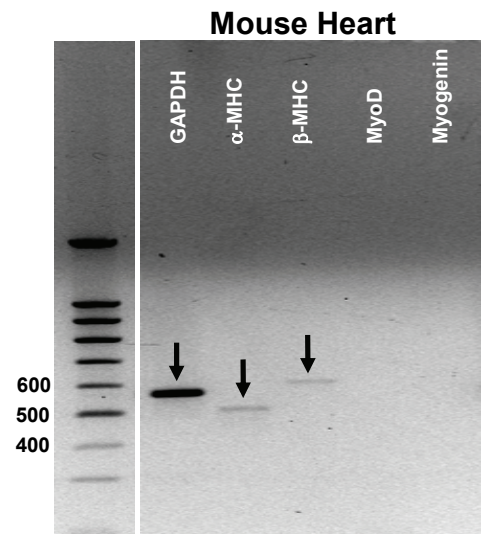

(c)

Figure 3 Expression of $\alpha-\mathrm{MHC}, \beta-\mathrm{MHC}$, MyoD and myogenin, as detected by nested-PCR, in (a) P19CL6 and $\mathrm{H} 9 \mathrm{C} 2(2-1)$ cells, cultured under adherent conditions, (b) P19CL6 cells cultured under non-adherent conditions, upon exposure to $1 \%$ DMSO, and (c) mouse heart tissue. Cells were cultured under adherent or non-adherent conditions for 15 days in the presence of $1 \%$ DMSO, as described under Methods. Heart tissue was dissected from 10 weeks old CD1 wild type $(+/+)$ male mice. Total RNA was isolated and mRNA levels determined by RT-PCR followed nested-PCR. Theoretical sizes of PCR products are $275 \mathrm{bp}$ or $413 \mathrm{bp}$ (GAPDH), 302 bp ( $\alpha$-MHC), 410 bp ( $\beta$-MHC), 392 bp (MyoD) and $337 \mathrm{bp}$ (myogenin), as indicated by the arrows. For Figure 3c the theoretical sizes of PCR products were: 556 bp (GAPDH); 491 bp ( $\alpha$-MHC); 587 bp ( $\beta$-MHC); 513 bp (MyoD); and 608 bp (Myogenin), as indicated by arrows. Data shown of one experiment that is representative of three separate biological experiments. 
tomes of mouse cardiac and skeletal muscle tissue, H9C2 (2-1) cells, and a reference sample, which was a mixture of cDNA from all the samples in equal proportions. An interwoven loop design and data reduction by linear modelling [21-23] was utilized to compare all samples to the reference sample (Figure 4). The transcriptomes of all samples were initially analysed by PCA and showed a low correlation between the P19CL6 cells and other samples (Table 2, Figure 5). Only the embryonic heart sample did not differ significantly from the P19CL6 cells $(\mathrm{P}>0.05)$, although even this comparison showed a very low correlation of 0.007 (Table 2). Subsequent PLS analysis, excluding the H9C2 (2-1) samples in order to simplify interpretation, showed that principal component 1 (PC1) accounted for $52 \%$ of the total variance and clearly separated the P19CL6 sample from the heart and muscle tissue (Figure 5). The loading factors for PC1 showing a variable importance (VIP) of $>1.0$ represent those transcripts driving the separation of P19CL6 cells from the other samples; these were put into a biological context by ascertaining Gene Ontology (GO) identifiers that were significantly over-represented in the identified transcript level changes, using the DAVID bioinformatics suite [24]. Such over-representation is often indicative of a significant biological effect in the pathway(s) associated with the GO identifiers. Several annotation clusters with an enrichment score of greater than 1.0, i.e. showing significant enrichment, were identified (502 separate genes) and the five main Biological Processes that were identified are shown in Table 3. A more detailed analysis of the genes identified within the "Regulation of cellular processes" cluster showed that 57\% (total number 146 separate gene products) of the identified mRNA levels were up-regulated in P19CL6 cells compared to embryonic heart tissue, whereas the remainder were downregulated (data not shown). The latter tissue was the primary focus for comparison as P19-derived cardiomyocytes are embryonic in nature and have previously been used as a model system for the embryonic heart $[1,6,8]$. However, in the original P19CL6 paper this cell-line was proposed to be a good model system for adult heart [9].

\section{DISCUSSION}

The P19CL6 cell-line, a derivative of P19 embryonal carcinoma cells, is widely used as an in vitro model of cardiovascular cells $[1,2,10,16]$, and has been shown to differentiate into a beating phenotype that is reminiscent of cardiomyocytes upon exposure to DMSO [9]. Data presented here confirm that differentiated P19CL6 cells do exhibit some markers of a cardiac phenotype in the P19CL6 cells: a beating phenotype that is positively responsive to adrenaline; expression of transcript markers of cardiac phenotype $(\alpha-\mathrm{MHC}, \beta-\mathrm{MHC})$; microscopic

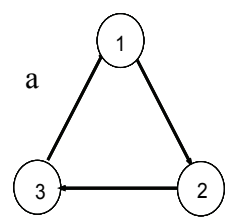

b
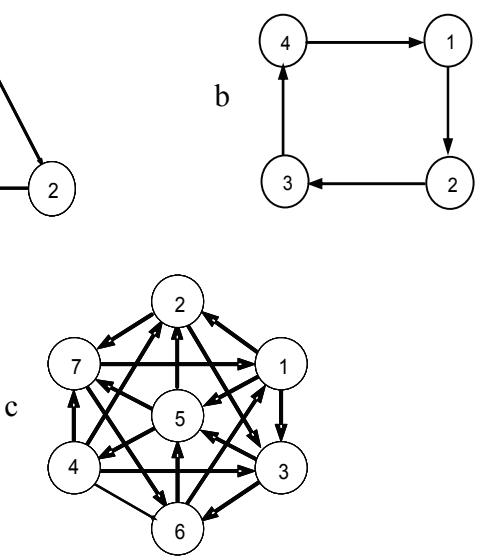

Figure 4. Representation of dual-hybridisation optimal interwoven loop design for microarray experiments. Loop designs showing three, four, and seven samples (nodes) that were compared in three different experiments. The samples analysed in each experiment were (a) P19CL6 cells, mouse embryonic heart and mouse adult heart tissue (b) P19CL6 cells, H9C2 (2-1) cells, mouse adult heart and mouse skeletal muscle tissue (c) P19CL6 cells (two different biological samples), H9C2 (2-1) cells, mouse embryonic heart tissue, mouse adult heart tissue, mouse skeletal muscle tissue and a reference sample, the latter containing cDNA from all the samples. The samples at the start of the arrows were labelled with $\mathrm{Cy} 3$ and the target samples with Cy5.

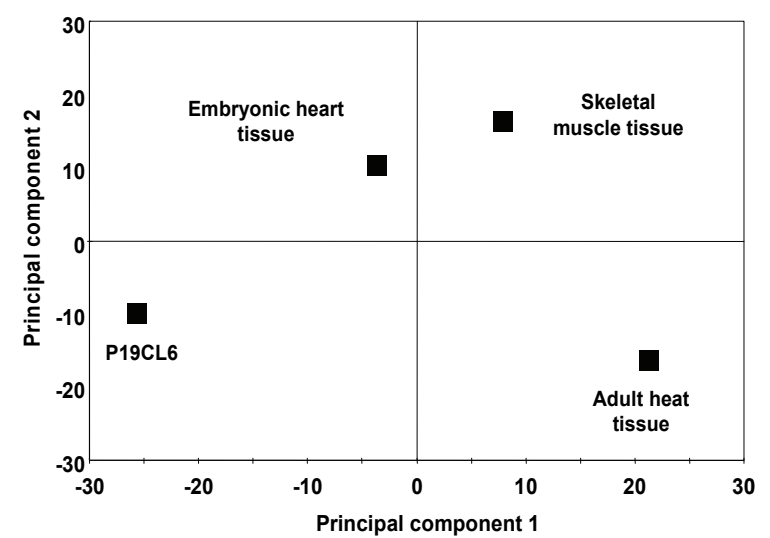

Figure 5. Analysis of transcriptomic expression data (microarrays) by PLS analysis. Gene expression data from microarray analysis was reduced by linear regression, using the LLAMA algorithm [19-21]. The data was further analysed by PCA and subsequently PLS to demonstrate discrimination between the samples $\left(\mathrm{R}^{2} \mathrm{X}\right.$ for principal components 1 and 2 is 0.52 and 0.33 , respectively) and generate a list of variable importance that could be used in the DAVID analysis.

analysis showing mono-nuclear cells with no evidence of cell fusion $[1,5,8,25]$. However, closer examination of these features raises some concerns, and is suggestive of an unstable, mixed, cardiac/skeletal muscle phenotype. We demonstrated that the pulse-rate for beating was negatively correlated with the passage number of the cells, with no beating observed after passage 16, suggesting 
Table 1. Sequences of oligonucleotide primers used for PCR.

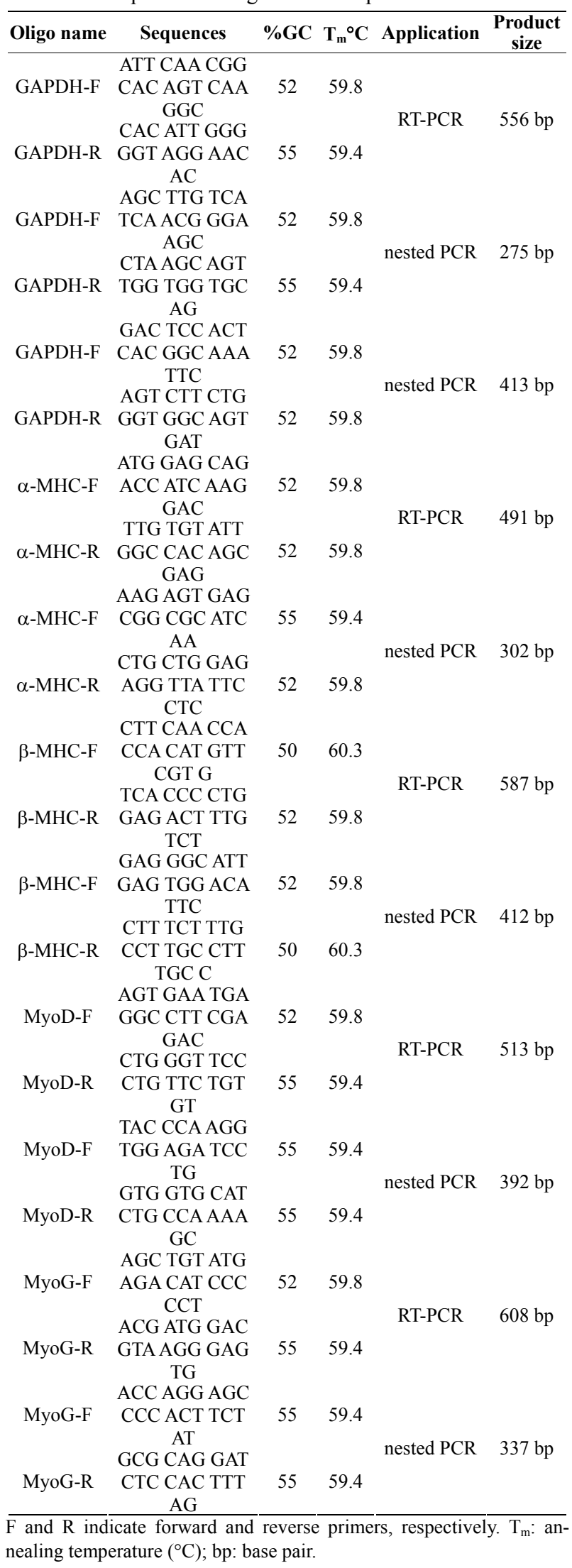

Table 2. Correlation matrix of mRNA expression levels in P19CL6 and H9C2 (2-1) cells, and skeletal muscle, adult heart and embryonic heart tissue samples, as analyzed by microarray analysis.

\begin{tabular}{ccccc}
\hline Samples & H9C2 (2-1) & $\begin{array}{c}\text { Skeletal } \\
\text { muscle }\end{array}$ & Adult heart & $\begin{array}{c}\text { Embryonic } \\
\text { heart }\end{array}$ \\
\hline P19CL6 & $-0.058^{*}$ & $-0.154^{* *}$ & $-0.210^{* *}$ & 0.007 \\
H9C2 (2-1) & -- & 0.031 & $0.175^{* *}$ & -0.023 \\
Skeletal muscle & -- & -- & $0.299^{* *}$ & -0.024 \\
Adult heart & -- & -- & -- & $0.373^{* *}$ \\
\hline
\end{tabular}

Microarray data was reduced by linear analysis using LLAMA and the data analyzed by PCA, with the data fitted to 5 components (explaining $100 \%$ of the cumulative variance). ${ }^{*} \mathrm{P}<0.05 ;{ }^{* *} \mathrm{P}<0.001$.

that the P19CL6 cells are subject to phenotypic drift with time in culture, and hence may not represent a stable cardiomyocyte phenotype. In addition, while P19CL6 cells expressed the aforementioned transcript markers of cardiac phenotype ( $\alpha-\mathrm{MHC}, \beta-\mathrm{MHC})$, they also expressed transcript markers for a skeletal muscle phenotype (MyoD and myogenin), again suggestive of a mixed cardiac/skeletal muscle phenotype. Whereas examination of single markers can give an indication of the potential phenotype for a cell-line they are not necessarily indicative of a fully functioning biological system. For example, liver cell-lines such as HepG2 have been validated for use in drug screening as hosts for both reporter genes and marker transcripts [26,27]; however, in-depth analysis demonstrates that this validation is simplified, with HepG2 cells unable to support some genome-based transcriptional activation and for the transcriptome to be markedly affected by culture conditions $[28,29]$. Therefore whereas low complexity measurements may be suitable for validation of cell-lines for use in individual assays, approaches such as transcriptome/proteome analysis are more appropriate to fully characterize celllines [30]; anchoring this data to known phenotypic markers then allows an accurate assessment of the appropriateness of any cell-line to the in vivo system they are supposed to be modelling [31]. Transcriptome analysis of P19CL6 cells demonstrated significant differences in the transcript profile between these cells and other samples, including importantly both embryonic and adult cardiac cells. Gene Ontology over-representation analysis suggests that these transcripts are linked to biological pathways associated with cellular metabolism, an interesting observation since it is generally accepted that cardiac muscle cells have specific metabolic processes that differentiate embryonic and adult cardiomyocytes, and also skeletal and cardiac muscle cells.

In conclusion, we have both undertaken physiological and transcriptome analysis of P19CL6 cells, assessing their suitability as models of cardiomyocytes for in vitro experimentation. Our data suggests that whereas the 
P19CL6 cell-line has some phenotypic similarities to cardiomyocytes (e.g. the ability to pulse) there exist significant differences between these cells and the in vivo situation. Our observations clearly demonstrate that the P19CL6 cell line does not maintain a robust cardiamyocyte phenotype as shown by the loss of cell beating with time in culture. In addition, transcriptome analysis clearly shows that even freshly differentiated cells do not exhibit a clear cardiac or muscle transcript profile, further questioning the utility of P19CL6 as a model system for the study of cardiomyocyte physiology.

\section{ACKNOWLEDGMENTS}

Technical advice and guidance on cDNA microarray work from Dr Giselda Bucca and Prof. Colin Smith, and Dr George Kass with microscopy, in the Faculty of Health and Medical Sciences, University of Surrey are gratefully acknowledged. Mr Ben Routley and Dr Mark Muldoon (The School of Mathematics, University of Manchester) provided valuable assistance in the use and interpretation of Live Linear Analysis of MicroArray (LLAMA) for analysis of microarray data. $\mathrm{Mr}$ Peter Kentish helped with the animal tissue studies. Financial support for this study was provided by the Iranian Ministry of Health and Medical Sciences and the Hamedan University of Medical Sciences.

\section{REFERENCES}

[1] Van der Heyden, M.A. and Defize, L.H. (2003) Twenty one years of P19 cells: what an embryonal carcinoma cell line taught us about cardiomyocyte differentiation. Cardiovascular Research, 58, 292-302.

[2] Anisimov, S.V., Tarasov, K.V., Riordon, D., Wobus, A.M., and Boheler, K.R. (2002) SAGE identification of differentiation responsive genes in $\mathrm{P} 19$ embryonic cells induced to form cardiomyocytes in vitro. Mechanisms of Development, 117, 25-74.

[3] McBurney, M.W., Jones-Villeneuve, E.M., Edwards, M.K. and Anderson, P.J. (1982) Control of muscle and neuronal differentiation in a cultured embryonal carcinoma cell line. Nature, 299, 165-167

[4] McBurney, M.W. (1993) P19 embryonal carcinoma cells. International Journal of Developmental Biology, 37, 135-140.

[5] Skerjanc, I.S. (1999) Cardiac and skeletal muscle development in P19 embryonal carcinoma cells. Trends Cardiovascular Medicine, 9, 139-143.

[6] Skerjanc, I.S., Petropoulos, H., Ridgeway, A.G. and Wilton, S. (1998) Myocyte enhancer factor $2 \mathrm{C}$ and Nkx2-5 up-regulate each other's expression and initiate cardiomyogenesis in P19 cells. Journal of Biological Chemistry, 273, 34904-34910.

[7] Wobus, A.M., Kleppisch, T., Maltsev, V. and Hescheler, J. (1994) Cardiomyocyte like cells differentiated in vitro from embryonic carcinoma cells P19 are characterized by functional expression of adrenoceptors and $\mathrm{Ca}^{2+}$ channels. In Vitro Cellular Development and Biology, 30A, 425-434.

[8] Rudnicki, M.A., Jackowski, G., Saggin, L. and McBurney, M.W. (1990) Actin and myosin expression during development of cardiac muscle from cultured embryonal car- cinoma cells. Developmental Biology, 138, 348-358.

[9] Habara-Ohkubo, A. (1996) Differentiation of beating cardiac muscle cells from a derivative of P19 embryonal carcinoma cells. Cell Structure and Function, 21, 101-110.

[10] Van der Heyden, M.A., van Kempen, M.J., Tsuji, Y., Rook, M.B., Jongsma, H.J. and Opthof, T. (2003) P19 embryonal carcinoma cells: a suitable model system for cardiac electrophysiological differentiation at the molecular and functional level. Cardiovascular Research, 58, 410-422.

[11] Eaton, S., Chatziandreou, I., Krywawych, S., Pen, S., Clayton, P.T. and Hussain, K. (2003) Short-chain 3-hydroxyacyl-CoA dehydrogenase deficiency associated with hyperinsulinism: a novel glucose-fatty acid cycle? Biochemical Society Transactions, 31, 1137-1139.

[12] Monzen, K., Hiroi, Y., Kudoh, S., Akazawa, H., Oka, T., Takimoto, E., Hayashi, D., Hosoda, T., Kawabata, M., Miyazono, K., Ishii, S., Yazaki, Y., Nagai, R. and Komuro, I. (2001) Smads, TAK1, and their common target ATF-2 play a critical role in cardiomyocyte differentiation. Journal of Cell Biology, 153, 687-698.

[13] Paquin, J., Danalache, B.A., Jankowski, M., McCann, S.M. and Gutkowska, J. (2002) Oxytocin induces differentiation of P19 embryonic stem cells to cardiomyocytes. Proceedings of the National Academy of Sciences USA, 99, 9550-9555.

[14] Peng, C.F., Wei, Y., Levsky, J.M., McDonald, T.V., Childs, G. and Kitsis, R.N. (2002) Microarray analysis of global changes in gene expression during cardiac myocyte differentiation. Physioogical Genomics, 9, 145-155.

[15] Ridgeway, A.G., Wilton, S. and Skerjanc, I.S. (2000). Myocyte enhancer factor $2 \mathrm{C}$ and myogenin up-regulate each other's expression and induce the development of skeletal muscle in P19 cells. Journal of Biological Chemistry, 275, 41-46.

[16] Young, D.A., Gavrilov, S., Pennington, C.J., Nuttall, R.K., Edwards, D.R., Kitsis, R.N. and Clark, I.M. (2004) Expression of metalloproteinases and inhibitors in the differentiation of P19CL6 cells into cardiac myocytes. Biochemical and Biophysical Research Communications, 322, 759-765.

[17] Morley, P. and Whitfield, J.F. (1993) The differentiation inducer, dimethyl sulfoxide, transiently increases the intracellular calcium ion concentration in various cell types. Journal of Cellular Physiology, 156, 219-225.

[18] Newton, C.A. and Graham, A. (1997) PCR. BIOS Scientific Publishers Ltd, Oxford.

[19] Goldsmith, Z.G. and Dhanasekaran, N. (2004) The microrevolution: applications and impacts of microarray technology on molecular biology and medicine. International Journal of Molecular Medicine, 13, 483-495.

[20] Vinciotti, V., Khanin, R., D'Alimonte, D., Liu, X., Cattini, N., Hotchkiss, G., Bucca, G., de Jesus, O., Rasaiyaah, J., Smith, C.P., Kellam, P. and Wit, E. (2005) An experimental evaluation of a loop versus a reference design for two-channel microarrays. Bioinformatics, 21, 492-501.

[21] Wit, E. and McClure, J. (2004) Statistics for microarrays: design, analysis, and inference. John Wiley \& Sons Ltd.. Chichester, United Kingdom.

[22] Dobbin, K. and Simon, R. (2002) Comparison of microarray designs for class comparison and class discovery. Bioinformatics, 18, 1438-1445. 
[23] Simon, R., Radmacher, M.D. and Dobbin, K. (2002) Design of studies using DNA microarrays. Genetic Epidemiology, 23, 21-36.

[24] Dennis, G., Sherman, B.T., Hosack, D.A., Yang, J., Gao, W., Lane, H.C. and Lempicki, R.A. (2003) DAVID: Database for Annotation, Visualization, and Integrated Discovery. Genome Biology, 4, 3.

[25] Rudnicki, M.A., Sawtell, N.M., Reuhl, K.R., Berg, R., Craig, J.C., Jardine, K., Lessard, J.L. and McBurney, M.W. (1990) Smooth muscle actin expression during P19 embryonal carcinoma differentiation in cell culture. Journal of Cellular Physiology, 142, 89-98.

[26] El-Sankary, W., Gibson, G.G., Ayrton, A. and Plant, N.J. (2001) Use of a reporter gene assay to predict and rank the potency and efficacy of CYP3A4 inducers. Drug $\mathrm{Me}$ tabolism \& Disposition, 29, 1-6.

[27] Morgan, K.T., Ni, H., Brown, H.R., Yoon, L., Qualls, C.W., Crosby, L.M., Reynolds, R., Gaskill, B., Anderson, S.P., Kepler, T.B., Brainard, T., Liv, N., Easton, M., Merrill, C., Creech, D., Sprenger, D., Conner, G., Johnson, P.R., Fox, T., Sartor, M., Richard,E., Kuruvilla, S., Casey, W. and Benavides, G. (2002) Application of cDNA mi croarray technology to in vitro toxicology and the selection of genes for a real-time RT-PCR based screen for oxidative stress in Hep G2 cells. Toxicologic Pathology, 30, 435-451.

[28] Morgan, K.T., Casey, W., Easton, M., Creech, D., Ni, H., Yoon, L., Anderson, S., Qualls, C.W., Crosby, L.M., MacPherson, A., Bloomfield, P. and Elston, T.C. (2003) Frequent sampling reveals dynamic responses by the transcriptome to routine media replacement in HepG2 cells. Toxicologic Pathology, 31, 448-461.

[29] Phillips, A., Hood, S.R., Gibson, G.G. and Plant, N.J. (2005) Impact of transcription factor profile and chromatin conformation on human hepatocyte CYP3A gene ex-

\section{ABBREVIATIONS}

ANOVA: Analysis of variance;

DMSO: Dimethyl sulphoxide;

RT-PCR: Reverse transcriptase polymerase chain reaction; pression. Drug Metabolism and Disposition, 33, 233-242.

[30] Plant, N. (2004) Strategies for using in vitro screens in drug metabolism. Drug Discovery Today, 9, 328-336.

[31] uo, W., Fan, W., Xie, H., Jing, L., Ricicki, E., Vouros, P., Zhao, L.P. and Zarbl, H. (2005) Phenotypic anchoring of global gene expression profiles induced by N-hydroxy-4 acetylaminobiphenyl and benzo[a]pyrene diol epoxide reveals correlations between expression profiles and mechanism of toxicity. Chemical Research in Toxicology, 18, 619-629.

[32] [Laderas, T. and McWeeney, S. (2007) Consensus framework for exploring microarray data using multiple clustering methods. OMICS, 11, 116-128

[33] Smyth, G.K. (2005) Linear Models and Empirical Bayes Methods for Assessing Differential Expression in Microarray Experiments. Statistical Applications in Genetics and Molecular Biology, 3, 1-26.

[34] Kimes, B.W. and Brandt, B.L. (1976) Properties of a clonal muscle cell line from rat heart. Experimental Cell Research, 98, 367-381.

[35] Buckingham, M. (2001) Skeletal muscle formation in vertebrates. Current Opinion in Genetics \& Development, 11, 440-448.

[36] Gulick, J., Subramaniam, A., Neumann, J. and Robbins, J. (1991) Isolation and characterization of the mouse cardiac myosin heavy chain genes. Journal of Biological Chemistry, 266, 9180-9185.

[37] Metzger, J.M., Lin, W.I., Johnston, R.A., Westfall, M.V. and Samuelson, L.C. (1995) Myosin heavy chain expression in contracting myocytes isolated during embryonic stem cell cardiogenesis. Circulation Research, $\mathbf{7 6}$, 710-719.

[38] Sabourin, L.A. and Rudnicki, M.A. (2000) The molecular regulation of myogenesis. Clinical genetics, 57, 16-25.
PCA: Principle components analysis; PLS: Partial least squares regression; LLAMA: Live Linear Analysis of MicroArray. 